Density of barley iSelect 50k SNP marker in the Barley Genome

chr2H

chr3H

$\mathrm{chr} 4 \mathrm{H}$

$\mathrm{chr} 5 \mathrm{H}$

chr6H

chr7H

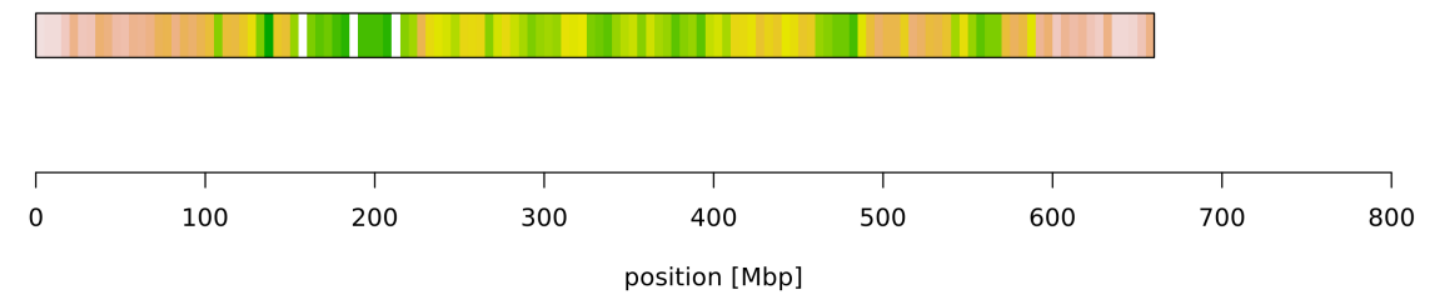

\section{Color scale}

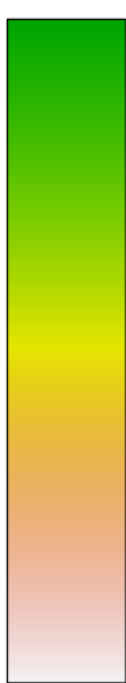

Max SNPs per 5 Mbp (372)

Min SNPs per 5 Mbp (0)

Figure S1. Distribution of markers on chromosomes. Colour indicates the density of markers per $5 \mathrm{Mbp}$. Decreasing marker density in the direction of telomeres can be observed. 